\title{
LHCb First Running Scenarios, Including Alignment and Calibration
}

\author{
G. Corti ${ }^{\mathrm{a}}$ (on behalf of the LHCb Collaboration) \\ ${ }^{\mathrm{a} C E R N,} 1211$ Genève, Switzerland
}

The running scenarios of the LHCb experiment in the early operation period of the LHC are discussed in view of the foreseen machine evolution. Aligment of the overall detector and its calibration will be the primary concern of the first runs; the strategy to perform them is described. The experiment aims to be in a stable running condition as soon as possible. Some of the measurements with the data collected in the first months of operation at a $14 \mathrm{TeV}$ collision energy are outlined.

\section{INTRODUCTION}

This paper presents the current running scenarios of the LHCb experiment in the early operation period of the Large Hadron Collider (LHC) at CERN. The primary goal of LHCb is to make precision measurements of $\mathrm{CP}$ violating decays and other rare phenomena in the $b$-system and so to make detailed tests of the Standard Model description of $\mathrm{CP}$ violation, as well as investigating possible new physics. A description of the experiment and its trigger can be found elsewhere in these proceedings $[1,2]$.

The objective of the earliest running period is to complete the commissioning of the experiment; this includes the alignment and calibration of the various sub-detectors and of $\mathrm{LHCb}$ as a whole. Tuning the triggers and understanding the experiment's performance are also important aspects of the first periods of data taking. LHCb is developing strategies for alignment, calibration and tuning of the trigger and analysis in view of the current start-up plans of the LHC, with the aim to exploit the physics potential of the experiment as soon as the physics running period of the LHC will start.

\section{COMMISSIONING STRATEGY}

The commissioning of the $\mathrm{LHCb}$ detector is first carried out without beam and will be completed when $p p$ collisions will occur in the experiment.

The first step has already started for some sub- detectors and consists of commissioning each of them individually, making sure specific hardware controls and monitors and safety systems are operational. In this phase, initial values of time delays are set and cabling verified, e.g. checking read-out and trigger connections. Most of these tasks are done locally using special portable commissioning racks. The different sub-detectors can be commissioned in parallel due to the partitioning of the Data Acquisition System (DAQ).

The global commissioning of LHCb will start in early 2007. One of the essential tasks to make the experiment ready for the first circulating beams will be the integration of the global Experiment Control System (ECS). The complete DAQ and error handling will also be commissioned at this time and beam-less calibration procedures will be verified. The synchronization of the Hadron Calorimeter (HCAL) and first level of trigger, Level-0, will be performed with light pulses in order to be able to collect events depositing energy in the HCAL as soon as beams will be present in the machine.

Although cosmic rays have been used for calibration and tests prior to installation they cannot be used to commission the $\mathrm{LHCb}$ detector as a whole because of the geometrical layout (shown in Fig.1) and due to the random arrival time of cosmic rays compared to the LHC clock to which the detector is synchronized. As a result, the commissioning of the experiment with its alignment and calibration will be completed when beams will circulate in the accelerator and collisions occur at 


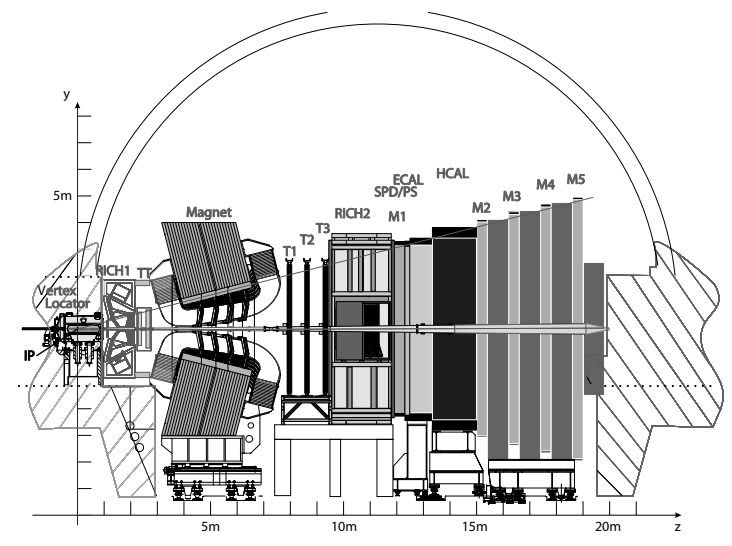

Figure 1. Side view of the LHCb detector; the interaction point is on the left with the Vertex Locator surrounding it.

the LHCb interaction point (IP).

\section{FIRST RUNNING SCENARIOS}

In the current official start-up plan, the LHC will begin operation at the end of 2007 with a Calibration Run with proton beams of energy $450 \mathrm{GeV}$. In 2008, the first Physics Runs at $14 \mathrm{TeV}$ collision energy will start after single beam commissioning at $7 \mathrm{TeV}$ and a period of collisions with a reduced number of bunches.

\subsection{Running scenarios with $450 \mathrm{GeV}$ proton beams}

The Calibration Run with proton beams of $450 \mathrm{GeV}$ and collisions at $900 \mathrm{GeV}$ will be very valuable in the commissioning of the detector. This period will allow operation of the complete detector with beams and to establish running procedures.

A reduced number of filled bunches per beam (43 in a first stage, 156 subsequently) will be circulated in the accelerator. Due to the displacement of the $\mathrm{LHCb}$ interaction point, only a fraction of these bunches, appropriately shifted, will collide at the IP, providing $\mathrm{LHCb}$ with a luminosity up to $\sim 1 \times 10^{27} \mathrm{~cm}^{-2} \mathrm{~s}^{-1}$ and a corresponding inelastic rate of $\sim 40 \mathrm{~Hz}$. Although the running conditions will be quite different from those expected in normal operation with reduced occupancies and data sizes, they will be quite favorable for both time and position alignment.

$\mathrm{LHCb}$ intends to also collect data in the earlier period of single-beam operation of the LHC. Beam-gas interactions upstream of the IP, in the Vertex Locator (VELO) region, and halo associated to the beam travelling from the IP to the Muon System, will provide particles useful for alignment very early on.

The alignment of the detector and establishing how to perform the tuning of the whole experiment in anticipation of the $14 \mathrm{TeV}$ collision energy will be the main concern of the running period during 2007. A special Level-0 trigger based on the HCAL energy deposition will be used.

\subsection{Time alignment}

The time synchronization of the $\mathrm{LHCb}$ detector as a whole requires particles traversing it. This is the first task that will be performed with beam present in the experiment.

Initial values of time delays will be set during the commissioning without beam and are expected to be internally coherent within subdetectors. In order to ensure the same event is read out by all sub-detectors, a special DAQ mode is used to record events selected by the HCAL Level-0 trigger together with the two previous and two successive potential bunch crossings, each separated by $25 \mathrm{~ns}$ time intervals. Due to the large spacing between filled bunches in the 2007 run, no activity is expected in the neighbouring (potential) crossing. Processing these consecutive events will allow each sub-detector to measure the time of the signal with respect to the time of the read-out and determine the best settings of each programmable delay in the system.

A fine-tune of the timing of the Muon System is essential to obtain the best Level-0 muon trigger efficiency; other methods are also considered for its alignment with data. The electronics channels of the Muon System will already be synchronized at the level of few nanoseconds with a dedicated pulse system during the commissioning phase in 2007, before circulating beams. 
As soon as the LHCb sub-detectors are synchronized, the DAQ will be switched to normal operation to acquire single bunch-crossing data. The next phase will be to align the positions of the detectors.

\subsection{Space alignment}

The overall alignment of the $\mathrm{LHCb}$ detector influences not only the final physics performance but also the High Level Triggers (HLTs) that rely on tracking and particle identification. The relative alignment of the entire detector, and in particular the Tracking System, is therefore one of the main objectives of the 2007 running period. It is of utmost importance to collect physics-quality data as soon as possible.

The LHCb Tracking System [3] is shown in Fig.1. It consists, from left to right, of a silicon strip detector (VELO) surrounding the IP. A set of silicon planes located $\sim 2.5 \mathrm{~m}$ downstream, referred to as Trigger Tracker (TT), are used in the trigger for a rough estimate of track momentum. Three tracking stations (T1-3), situated after the dipole magnet $(\sim 7.5-9.5$ meters from the IP), each comprise of a silicon-strip based Inner Tracker (IT) and a straw-tube based Outer Tracker (OT).

At installation time, the position of all detectors will be surveyed to different precisions; for the tracking system it is expected to achieve measurement precisions of $0.3-0.5 \mathrm{~mm}$.

The LHCb alignment strategy [4] consists of first determining the internal alignment constant of each detection plane. The hits produced by charged particles are used to independently reconstruct segments of tracks in the VELO and the T-Stations. These charged-track segments are used to internally align, in parallel, the VELO, the IT, the OT, and IT-to-OT. The internal alignment technique chosen for the tracking detectors is a non-iterative method using the matrix inversion program Millepede [5]. In this approach, the trajectories are expressed as a linear combination of local (track dependent) and global (alignment) parameters. The parameters are then obtained via the minimization of a $\chi^{2}$ function.

The VELO is the most precise device in $\mathrm{LHCb}$ and an integral part of the trigger. The VELO silicon planes move into (out of) the beam during collision (injection). The nominal location of the first silicon sensor strip is at a radius of $8.16 \mathrm{~mm}$ from the beam line. Prior to the LHC establishing stable beams, the VELO is retracted by $\sim 3 \mathrm{~cm}$ and brought step-wise to its nominal position only when stable beams are declared and the luminous region determined. This operation will occur between each fill of the LHC and should become an automated and routine operation; this is one of the commissioning tasks of the 2007 run. In 2007 the VELO will be first internally aligned in its open position and also the luminous region will be determined. The final alignment of the VELO will then be done when in its closed position. The internal software alignment precision for the final setup is expected to be of the order of $2 \mu \mathrm{m}$. The VELO alignment has been validated in a set of Alignment Challenge and Detector Commissioning test-beam runs that took place in recent months.

The alignment of the T-Stations to the VELO will be performed by comparing segments at the center of the magnet and is expected to have a precision of the order of $20 \mu \mathrm{m}$. Once the VELO and T-Stations are brought into relative alignment, the TT will be aligned by using residuals of TT hits to the tracks reconstructed with the VELO and T-Stations.

The tracking detectors are aligned by requiring that the hits produced by charged particles lie along a trajectory, assumed to be known. When magnetic field is present the trajectory is curved and the predicted position of a particle at a given location will depend on the knowledge of the momentum and of the magnetic field. To remove this bias, the full geometrical alignment of the tracking system will be first performed with the $\mathrm{LHCb}$ dipole magnet switched off and using the Calorimeters to provide an energy measurement of each track. The alignment of the Tracking System will then be adjusted once the magnetic field is brought to its nominal value. This strategy fits well with LHC start-up in 2007 when the dipole is expected to be initially switched off. The possibility of the VELO operating with magnetic field on during the $450 \mathrm{GeV}$ run is under investigation, given the special layout of the machine optics. 
Once the Tracking System is aligned, the other detectors will be aligned with respect to it. The two RICH detectors are most sensitive to misalignment since knowledge of the track vector contributes to the Cherenkov angle resolution. A number of different components will need to be aligned. The RICH Hybrid Photon Detectors (HPDs) are first aligned using a stand-alone calibration system, currently being designed. The mirror systems of the RICHes are aligned by comparing the reconstructed photon positions with those expected (based on the charged track parameters).

Accurate measurements of the Electromagnetic and Hadron Calorimeters (ECAL, HCAL) positioning have been achieved during their installation; the relative position of the ECAL modules both in the directions perpendicular to the beams is within $\pm 0.5 \mathrm{~mm}$. The Muon System is currently being installed and a position accuracy of $\sim 1 \mathrm{~mm}$ is expected. The final alignment of the ECAL and the Muon System to the Tracking System will be performed with clean electron and muon samples.

\subsection{Running scenarios with $7 \mathrm{TeV}$ proton beams}

The operation of the LHC in 2008 will proceed in stages. First the $7 \mathrm{TeV}$ beams will be commissioned; after this period of single-beam operation there will be collisions at $14 \mathrm{TeV}$ energy with a reduced number of filled bunches, similar to the $450 \mathrm{GeV}$ Calibration Run. A second stage of operation with $75 \mathrm{~ns}$ spaced bunches will follow. Finally, a third stage with $25 \mathrm{~ns}$ spaced bunches is expected, as in the nominal LHC.

The luminosity in the $\mathrm{LHCb}$ IP is expected to increase from $\sim 1 \times 10^{28} \mathrm{~cm}^{-2} \mathrm{~s}^{-1}$ to $1.2 \times$ $10^{32} \mathrm{~cm}^{-2} \mathrm{~s}^{-1}$, depending on the operating conditions. This almost reaches the experiment design luminosity of $\sim 2 \times 10^{32} \mathrm{~cm}^{-2} \mathrm{~s}^{-1}$.

We intend to complete the calibrations of $\mathrm{LHCb}$ energy, momentum and particle identification in the first period of LHC operation at $14 \mathrm{TeV}$. Completing the commissioning of the Level-0 and HLTs to their design output rates will also be one of the main objectives.

The purpose of the $\mathrm{LHCb}$ running program in
2008 will be to achieve stable running operation as soon as possible and collect physics-quality data to produce first results.

\subsection{Momentum and energy calibration}

The momentum resolution is sensitive to both misalignment of the Tracking System and uncertainties in the knowledge of the magnetic field. The experiment plans regular flipping of the polarity of the $B$ field to better understand detectorinduced asymmetries. The $B$ field components were measured at the end of 2005 in the area of the Tracking System (from the VELO to the Tstations); magnetic field maps for both polarities have been produced. The $B$ field is known with $\leq 0.03 \%$ uncertainty. This affects less than $10 \%$ the intended momentum resolution of $\delta p / p=$ $(0.35-0.55) \%$. The momentum scale will be verified and adjusted via the mass peaks of the $K_{S}^{0}$, $J / \Psi, \Upsilon, Z^{0}$, etc.

Different methods for the energy calibration of the ECAL have been investigated and a procedure of successive alternative steps is envisaged. The use of cosmic rays prior to installation has allowed a calibration of the modules at the $10 \%$ level. Monte Carlo studies have shown that an energy-flow method will then calibrate the modules at the level of a few \%. Finally, an iterative procedure on the mass of resolved $\pi^{0} \mathrm{~s}$ will bring the energy calibration to $1 \%$ [6].

\subsection{Tuning the Triggers}

The Level-0 trigger of $\mathrm{LHCb}$ is a hardware trigger based on high $E_{t} / p_{t}$ particles $\left(\mu, e, h, \pi^{0}, \gamma\right)$. Its commissioning will start during data taking with $450 \mathrm{GeV}$ protons and the output rate will be tuned to $1 \mathrm{MHz}$ on the onset of $14 \mathrm{TeV}$ collisions. The HLTs are software triggers based on high $E_{t} / p_{t}$ particles, impact parameter, displaced vertices, $B$ particles masses, etc. The HLTs comprise several streams to refine the Level-0 trigger; inclusive and exclusive selections. The commissioning of the HLT will also start in the 2007 running period with the tuning of the on-line tracking and will be completed in early 2008, tuning the output rate to $2 \mathrm{kHz}$. A detailed description of the $\mathrm{LHCb}$ triggers can be found elsewhere in these proceedings [2]. 


\subsection{Particle identification}

$\mathrm{LHCb}$ has two RICH detectors for particle identification (PID) over different momentum ranges. They will be calibrated first via basic measurements: saturated tracks collected with the magnetic field switched off will provide Cherenkov angle resolution while those collected with the $B$ field on will allow calculation of the radiator refractive indices. The $\mathrm{RICH}$ internal alignment is also an important component to the Cherenkov angle resolution; its contribution is aimed to be kept below $0.1 \mathrm{mrad}$, which is to be compared to $1.27 \mathrm{mrad}$ per photo-electron in RICH1, which includes the effect of tracking resolution.

A special data sample will also be used to calibrate the RICH system and evaluate its performance independently of Monte Carlo simulation. The $D^{*} \rightarrow D^{0}(\rightarrow K \pi) \pi$ decay chain provides a clean sample of $K$ and $\pi$ at well-defined momenta, due to the constrained kinematics of the decay. A HLT stream is dedicated to recording these events. Details on the RICH system calibration procedure using this technique can be found elsewhere in these proceedings [7].

\section{PHYSICS PROGRAM}

An integrated luminosity of $0.5 \mathrm{fb}^{-1}$ is expected to be collected in the first year of LHC operation at nominal energy. The LHCb physics program with these very first data includes production studies of charmonium in a region of phase space complementary to that of the other LHC experiments. $\mathrm{LHCb}$ will record a very large unbiased sample of $J / \Psi$ from the $600 \mathrm{~Hz}$ HLT inclusive dimuon stream. For example this sample will allow a measurement of the ratio of prompt $J / \Psi$ to $B \rightarrow J / \Psi$ production.

The measurement of the angle $\beta$ with $B^{0} \rightarrow$ $J / \Psi K_{S}^{0}$ will be one of the first LHCb measurements of $\mathrm{CP}$ violation. With an integrated luminosity of $0.5 \mathrm{fb}^{-1}$, a statistical uncertainty on $\sin (2 \beta)$ of $\sim 0.04$ has been estimated [3].

The observation of a large CP asymmetry in $B_{s}^{0} \rightarrow J / \Phi \phi$ would be a striking signal for physics beyond the Standard Model (SM). With the expected statistical sensitivity of the data collected in the 2008 Physics Run, the experiment will either set a limit on, or measure, new physics enhancements [3].

The physics program with the first data also includes the $B_{s}^{0} \rightarrow \mu \mu$ decay that, suppressed in the $\mathrm{SM}\left(B R \sim 3.5 \times 10^{-9}\right)$, can be strongly enhanced in SUSY models. Recent preliminary studies show that LHCb could already exclude branching ratios down to the SM prediction with the luminosity expected in 2008 [8].

\section{CONCLUSIONS}

A detailed LHCb commissioning strategy is being prepared. The strategy for calibration, alignment, tuning of the triggers and of the analysis is being devised in view of the current LHC startup schedule. LHCb plans to perform the commissioning of the detector with beam during the LHC Calibration Run in 2007 and, in order to achieve stable running operation as soon as possible, to complete commissioning in the early running period of 2008. Interesting physics measurement will be possible with the $0.5 \mathrm{fb}^{-1}$ integrated luminosity expected in the first year of stable operation at $14 \mathrm{TeV}$.

\section{REFERENCES}

1. Ll. Garrido, these proceedings.

2. E. Rodriguez, these proceedings.

3. R. Antunes Nobrega et al., CERN-LHCC2003-030 (2003).

4. W. Baldini et al., LHCb-2006-035 (2006).

5. V. Blobel and C. Kleinwort, hep-ph/020821

6. K. Voronchev and I. Belyaev, LHCb-2006-051

7. R. Muresan, these proceedings.

8. F. Teubert, Proceedings of "Flavour in the era of the LHC Workshop" (to be published). 